\title{
Paper-based platforms with coulometric readout for ascorbic acid determination in fruit juices $†$
}

\author{
Estefanía Núnez-Bajo and M. Teresa Fernández-Abedul (iD *
}

There is growing interest in the development of simple, fast, sustainable and low-cost analytical methodologies on paper-based platforms. However, sensitive detection strategies that fit properly with these devices are still required. In this work, a calibration-free method is proposed for analytical determinations performed on paper-based electrochemical devices, in this case, for ascorbic acid. Carbon ink is deposited on a hydrophilic working area of the paper delimited with a hydrophobic wax. This maskless procedure is fast and cuts down ink waste. The connection of this working electrode to the potentiostat is 20 provided by reusable gold-plated connector headers that provide also the reference and counter electrodes. The thickness of the paper substrate defines the electrochemical cell and confines a sample volume, ideal for thin-layer coulometry. Controlled-potential coulometry is performed applying a potential of $+0.6 \mathrm{~V}$ for $50 \mathrm{~s}$. The charge is calculated by measuring the area under the fast chronoamperogram and the concentration is determined following Faraday's law (known number of transferred electrons). This methodology was applied to the determination of ascorbic acid, with a limit of detection of $40 \mu \mathrm{M}$. Its concentration in commercial fruit juices can be directly determined in diluted samples. The absence of matrix eff ects is observed by comparing the results obtained before and after the enzymatic reaction

Received 9th March 2020, of

Accepted 11th March 2020

the sample with cucumber ascorbate oxidase. Good accuracy and precision makes this method suitable

DOI: $10.1039 / d 0 a n 00477 d$ for quality control of ascorbic acid in commercial juices. Underexploited coulometric readout can be applied as a fast (calibration-free) and low-cost (standards not required) transduction principle to the rsc.li/analyst newly developed paper devices.

\section{Introduction}

Paper-based analytical devices are an excellent example of platforms extremely useful for decentralized analysis. After the development of sensitive and selective methodologies based on the use of sophisticated instruments, their miniaturization together with the simplification of the procedures has paved the way for the decentralization of analysis, a new milestone in analytical chemistry. Low-cost materials, portable equipment and easy-to-perform protocols are mandatory requirements for this type of analysis. In this context, paper-based electrochemical platforms are gaining attention, ${ }^{1,2}$ and diff erent techniques, mainly amperometry and potentiometry, are employed. ${ }^{3-5}$ However, coulometric readout is an under50 exploited alternative that fits perfectly with low-cost, userfriendly and fast-response devices. Moreover, this is an absol-

\footnotetext{
Departamento de Química Física y Analítica, Universidad de Oviedo, Asturias, Spain. E-mail: mtfernandeza@uniovi.es; Tel: +34 (9)85102968

† Electronic supplementary information (ESI) available: Influence of the carbon ink concentration on the sensitivity; chronoamperograms of the calibration curve; LSV recorded in enzymatically-treated and untreated fruit juice samples. See DOI: $10.1039 / \mathrm{d} 0 \mathrm{an} 00477 \mathrm{~d}$
}

ute electrochemical technique that does not require calibration if $100 \%$ of the charge is employed in the electrolysis of the analyte/indicator molecule. In addition, it is not aff ected by solvent evaporation since it depends on the number of moles of the analyte, not on its concentration. The analytical signal (charge that passes through the working electrode, $Q$ ) and the number of moles of electroactive species $(N)$ are related through Faraday's law $(Q=n F N)$, with a constant factor that is the number of electrons multiplied by the Faraday constant $(F)$. If the number of electrons is known, the charge required to electrolyze the analyte (or a related species) in a sample can be directly related to the number of moles.

Then, coulometry (e.g., controlled-potential coulometry) is a powerful tool in electroanalysis due to its ability to directly derive the quantity (number of moles) or electron stoichiometry $(n)$ of a substance without the use of a calibration curve. ${ }^{6,7}$ Since exhaustive electrolysis has to be ensured, faster methodologies are achieved at high surface/volume ratios and under eff ective mass-transfer conditions. The basis of thinlayer coulometry at metal electrodes was long described. ${ }^{8}$ It has been demonstrated that the establishment of thin-layer samples allows one to impose the total conversion/depletion of the species of interest once an appropriate driving force (e.g. 
1 constant or linear sweep potential) is applied to the system. ${ }^{9,10}$ If the sample level of the analyte can be quantitatively and selectively depleted by the sensor, the current may be integrated to give the total charge accumulated and then the amount of the analyte. To reduce the analysis time, the sample layer thickness should normally not exceed that of the diff usion layer (typically less than $100 \mu \mathrm{m}) .{ }^{9}$ If the entire current can be ascribed to the reaction of interest (excellent selectivity and nearly $100 \%$ coulometric efficiency) and the

sample volume is known, one may achieve a calibration-free (bio)sensor. ${ }^{10}$

Although many key characteristics of paper have been considered so far in the development of analytical devices since Whitesides'11 and Henry's ${ }^{12}$ groups developed the first colorimetric and electrochemical (amperometric) devices on paper substrates, the coulometric readout has not been sufficiently exploited. However, the combination of confined thin-layer samples with volumes on the order of a few microliters or less

in paper substrates would off er exhaustive analyte consumption, resulting in an ideal calibration-free methodology that would become truly useful in field situations. Recently, a Nafion® membrane was used to separate two half-cells with silver-foil electrodes as the working and reference (anodized in

chloride medium to generate $\mathrm{AgCl}$ ) electrodes. Two pieces of paper were located over the foils to perform thin-layer coulometry for the detection of multiple halides by the application of linear potential scans. Coulometric readout is performed using cyclic voltammetry as the interrogation technique and calibration curves are obtained representing the charge (calculated by measuring the area under the voltammogram) and the halide concentration. ${ }^{9}$ On the other hand, the working electrode can be incorporated in the porous paper substrate by using conductive suspensions, which allowed performing controlled-potential coulometry for enzymatic determination of glucose. ${ }^{13}$

Ascorbic acid (AA) or vitamin $\mathrm{C}$ is one of the most important vitamins in the human diet since it is involved in various biochemical pathways and acts as a powerful antioxidant. Then, it is important to monitor AA levels in dietary sources (fruits and vegetables, food supplements, and vitamin formulations) as well as in biological liquids, such as urine and serum. As an electron donor, ascorbic acid serves as one of the

45 most important low-molecular-weight antioxidants in the food industry to prevent unwanted changes in color or flavor. It contributes to the total antioxidant capacity, an important quality indicator of foods and drinks. ${ }^{14}$ Due to the crucial role of vitamin $\mathrm{C}$ in biochemistry and industrial applications, its

50 determination attracts high interest. Rapid monitoring of vitamin $\mathrm{C}$ levels during production and quality control stages is also important.

Determination of AA includes electrochemical ${ }^{15,16,17-22}$ and spectrophotometric methods (with absorbance, ${ }^{23,24}$ fluorescence ${ }^{25}$ or chemiluminescence ${ }^{26}$ measurements). Electrochemical detection is low-cost, easily miniaturizable, simple and fast, characteristics that are very convenient for decentralized analysis. Although several electrochemical methods have been developed for AA determination using 1 unmodified $^{18}$ or modified ${ }^{15,16,21}$ electrodes, direct coulometry has not been further exploited. Related works deal with indirect coulometry (coulometric titrations), which requires the coulometric generation of the titrant agent (e.g., iodine, ${ }^{27} 5$ employed for validation of an amperometric methodology developed for mapping AA in oranges). A PDMS multicompartment electrolysis cell has been designed for performing coulometric titrations of acetic and ascorbic acids in vinegar and vitamin $\mathrm{C}$ dietary supplement tablets. ${ }^{28}$ Endpoints of the titra- 10 tions were determined from the visual color change of an indicator. Moreover, extensive electrolysis has been employed in an origami paper device for complete elimination of interferents (ascorbate, urate and paracetamol) of a glucose enzymatic electrochemical sensor, but further detection is performed amperometrically. ${ }^{29}$

Therefore, an electrochemical calibration-free method is very promising for in situ control of AA levels in foodstuff $s$. In

this work, the development of a controlled-potential coulo- 20 metric method on paper-based devices for ascorbic acid determination is presented for the first time. The sample diff uses from the surface of the paper to the working electrode, placed at the bottom of the electrochemical cell, by capillary action. Since it is confined to the cavities/pores of the paper, the 25 sample can be considered as a thin film that is in direct contact with the working electrode, a paper-based carbon working electrode (PCWE). Gold-plated connector headers are coupled to the PCWE, providing stable reference and counter electrodes as well as connection to the potentiostat. As a proof 30 of concept, the coulometric method was applied to the determination of AA in fruit juices, opening the possibility of decentralized determination of many analytes of interest, not only in food but also in, e.g., the environmental, clinical, pharmaceutical and industrial fields.

\section{Experimental}

\section{Materials and methods}

Reagents and materials. L-Ascorbic acid (AA), sodium acetate and dimethylformamide (DMF) were purchased from SigmaAldrich (USA). Sodium hydroxide and acetic acid (100\%) were supplied by Merck. All the solutions were prepared in Milli-Q 45 water (Merck Millipore). Orange, apple and multifruit juice samples as well as cucumber were purchased from a local grocery (Asturias, Spain).

Whatman chromatographic filter paper Grade 1 was obtained from GE Healthcare Life Sciences (UK). Hydrophobic 50 areas were printed with a wax printer model ColorQube 8570 DS (Xerox, USA) controlled by Adobe Illustrator CC software. Transparency sheets were acquired from local stationeries. Carbon ink was manufactured by Gwent (UK).

Preparation of paper-based carbon electrodes. The Whatman Grade 1 paper (180 $\mu \mathrm{m}$ thick) was wax-printed with a pattern designed with Adobe Illustrator CC software. The pattern consisted of $7 \mathrm{~mm}$ diameter empty circles on the back- 

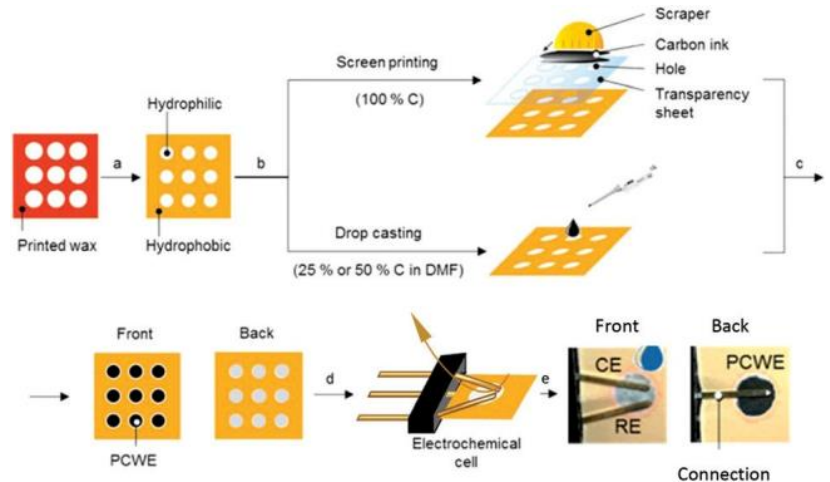

Fig. 1 Schematic representation of the fabrication of paper-based carbon working electrodes (PCWEs) and a complete electrochemical cell: (a) Wax melting at $110^{\circ} \mathrm{C}$ for 2 min after wax printing; (b) electrode fabrication; (c) curing process at $70^{\circ} \mathrm{C}$ for $1 \mathrm{~h}$; (d) coupling of the PCWE with golden-plated connector headers, the carbon ink is at the bottom of the electrochemical cell; (e) addition of solutions on the electrochemical cell.

ground of the wax. Hydrophobic barriers were created by wax melting in a hot plate at $110^{\circ} \mathrm{C}$ for $2 \mathrm{~min}$ (Fig. 1a), which generated $4 \mathrm{~mm}$ diameter hydrophilic circles. Working electrodes 25 (carbon ink on paper) as well as auxiliary and reference electrodes (gold-plated pins of a commercial connector) were deposited (in the case of the ink) or clipped to paper (wire electrodes) to complete the electrochemical cell, as indicated below. Depending on the carbon ink concentration, screenprinting ( $100 \% \mathrm{C}$ ink) or drop-casting ( 25 and $50 \% \mathrm{C}$ ink) procedures were carried out (Fig. 1b). The viscosity of the ink does not allow using micropipettes when a $100 \%$ carbon ink is employed. In this case a screen-printing procedure is employed. With this aim, $4 \mathrm{~mm}$ holes were cut on a transparency sheet with a paper punch and $100 \%$ carbon ink was printed through the mask. Drop casting was performed on each hydrophilic circle by dropping $2 \mu \mathrm{L}$ of $25 \%$ or $50 \%$ carbon ink suspensions in dimethylformamide (DMF). When

the ink solution is deposited on the paper (at the center, approximately), two phenomena could be observed: (i) a radial dispersion that happens by capillarity, where the solvent could wet the hydrophobic wax. In some cases a small circumference can be noticed surrounding the working area, and (ii) the

45 carbon ink remained in the hydrophilic circle, slightly separated from the circular wax borders. This can be perceived as a white crown that surrounds the ink. Both phenomena, which do not aff ect measurements, can be seen in Fig. 1e.

After solvent evaporation for $1 \mathrm{~h}$ at $70{ }^{\circ} \mathrm{C}$, paper-based 50 carbon electrodes (Fig. 1c) were cut and coupled to gold-plated connector headers as shown in Fig. 1d. The paper layer was inserted between three gold-plated pins in such a way that the one in the middle acted as a connection for the working electrode (WE) and the other two were located at the opposite side

of the paper to avoid short circuits between electrodes. ${ }^{30}$ The separation from the plane forms a clip that holds the paper. Finally, $6 \mu \mathrm{L}$ of solutions were added on the side where the reference (RE) and counter (CE) electrodes are located (Fig. 1e).
Equipment and measurements. Cyclic voltammetry (CV), linear-sweep voltammetry (LSV) and chronoamperometry (CA) were performed using a portable potentiostat model $\mu$ STAT 300 provided by DropSens (Spain) and controlled using DropView 8400 software.

Cyclic voltammetry (CV) was performed in $1 \mathrm{mM}$ AA solution in $0.1 \mathrm{M}$ acetate buff er (AB) $\mathrm{pH} 3.5$ using paper-based working electrodes made of $25 \%$ carbon suspension. The potential was swept from -0.4 to $+1.0 \mathrm{~V} v$ s. gold pseudo-reference electrode at a scan rate of $100 \mathrm{mV} \mathrm{s}^{-1}$.

Coulometric determination of AA was carried out integrating the current of the amperometric signals obtained by linear sweep voltammetry (LSV) and chronoamperometry (CA). In the first case, the potential was swept from +0.0 to $+1.0 \mathrm{~V} v s$. gold pseudo-reference electrode at $100 \mathrm{mV} \mathrm{s}^{-1}$. The charge $(\mu \mathrm{C}$ or $\mu \mathrm{As}$ ) was calculated by dividing the area under the LSV curve, $A_{\mathrm{LSV}}(\mu \mathrm{A} \mathrm{mV})$, by the scan rate, $\mathrm{V}\left(\mathrm{mV} \mathrm{s}^{-1}\right)$. Chronoamperometry was performed in two successive steps:

(i) a potential of $+0.0 \mathrm{~V} v s$. gold pseudo-reference electrode was applied for $10 \mathrm{~s}$ to get a stable baseline and (ii) AA was electro-

lyzed at $+0.6 \mathrm{~V} v s$. gold pseudo-reference electrode for $60 \mathrm{~s}$. The area under the chronoamperogram $(\mu \mathrm{A} s)$ proportionated the charge directly.

All the electrochemical measurements were performed in 25 triplicate, using diff erent paper substrates (PCWEs).

Sample preparation and determination of ascorbic acid in real samples. To investigate possible matrix interferences in coulometric and amperometric methodologies, selective enzymatic elimination of ascorbic acid was accomplished by using 30 cucumber, ${ }^{31}$ Cucumis sativus, a natural source of the enzyme ascorbate oxidase. The peel of a cucumber was grated and an optimized amount was added to $1.0 \mathrm{~mL}$ of the sample juice (pH 5-6) or to $0.5 \mathrm{mM} \mathrm{AA}$ (concentration close to that present in orange juices) solution in acetate buff er pH 5.0. Mixtures 35 were kept under vortex stirring for $15 \mathrm{~min}$ at room temperature and the supernatant was diluted with $1.0 \mathrm{~mL}$ of acetate buff er $\mathrm{pH}$ 3.5. Coulometric determination of AA in real samples was performed and chronoamperograms were recorded before and 4 after the enzymatic pretreatment (as described in the previous section). The concentration of AA in the sample was calculated from the diff erence between the amounts obtained by applying Faraday's law to charges measured before and after the enzymatic treatment.

\section{Results and discussion}

Voltammetric study of the process of $\mathrm{L}_{\text {-ascorbic acid }}$

The electrochemical behavior of AA on PCWEs was studied and the influence of the $\mathrm{pH}$ on the electrochemical signal and the driving force (in this case, the potential) required for the total depletion of the analyte were first studied.

Ascorbic acid is an electroactive biological compound that 55 is easily oxidized. Due to its low stability, AA is rapidly oxidized (Scheme S1†) to dehydroascorbic acid (DHA). This can be induced by enzymes such as ascorbate oxidase, oxygen, alka- 
1 line $\mathrm{pH}$, hydroxide ions, high temperature, the presence of metals (copper and iron) or exposure to light and radiation. ${ }^{32,33,34,35}$ The electrochemical oxidation of ascorbic acid (AA) was further investigated on conventional electrodes. ${ }^{36,37,38,39}$ Ascorbic acid undergoes an irreversible process (oxidation) in aqueous solution above $\mathrm{pH} 4.0$ at room temperature.

Initial studies were performed to investigate the electrochemical behavior of AA at electrodes fabricated by depositing 10

$25 \%$ carbon ink suspensions on Whatman Grade 1 filter paper. A recent article $^{2}$ reviews past works related to screenprinted electrodes, but also to stencil-printed, inkjet-printed, and pencil/pen-drawn electrodes as well as to the use of microwires or electrodes made following other procedures (e.g. sputtering). However, we consider the drop-casting method as very advantageous compared to screen-printing because the use of masks is not required and smaller amount of carbon is spent, which reduces the cost and time of analysis.

Cyclic voltammograms recorded in $1 \mathrm{mM}$ AA solution in acetate buff er of $\mathrm{pH} 3.5$ are shown in Fig. S1(a). $\dagger$ A well-

defined and precise anodic process was obtained on PCWEs at $+0.43 \pm 0.04 \mathrm{~V} v s$. gold pseudo-reference electrode, with a peak current of $21.7 \pm 0.5 \mu \mathrm{A}$. The anodic process shifted slightly toward less positive potentials with increasing $\mathrm{pH}$ (from +0.43 $\pm 0.04 \mathrm{~V}$ at $\mathrm{pH} 3.5$ to $+0.31 \pm 0.05 \mathrm{~V}$ at $\mathrm{pH} 5.5 \mathrm{vs}$. gold pseudoreference electrode, Fig. $\mathrm{S} 1(\mathrm{~b}), \dagger$ demonstrating that protons participated in the electrochemical reaction. The potential shift is in consonance with previous reports. ${ }^{40}$ Lower currents were obtained as the $\mathrm{pH}$ increased, with anodic peak currents that changed from $20.2 \pm 0.6 \mu \mathrm{A}(\mathrm{pH} 3.5)$ to $0.4 \pm 0.1 \mu \mathrm{A}(\mathrm{pH}$ 5.5). A pH value of 3.5 was chosen for the rest of the electrochemical measurements.

Having selected $\mathrm{pH} 3.5$ as the optimum value for further studies, the scan rate dependence of the anodic peak current was evaluated for scan rates ranging from 10 to $200 \mathrm{mV} \mathrm{s}^{-1}$. Previous works related to the AA determination on unmodified carbon electrodes reported that the anodic oxidation peak of AA has a direct dependence on the square root of the scan rate, ${ }^{37,38,39-41}$ according to the Randles-Sevcik equation for a flat electrode and diff usion-controlled processes at $25{ }^{\circ} \mathrm{C} .{ }^{27,42}$ Fig. 2A shows the LSV responses and the logarithmic plot of the peak current vs. the scan rate. The slope of the line in Fig. 2B (inset) is $0.59 \pm 0.01\left(R^{2}=0.998\right)$, which does not correspond to the value of 1 expected for adsorbed electroactive species, neither to the 0.5 associated with the semi-infinite diff usion of the electroactive species to the electrode. A similar behavior was observed for the detection of nicotine on pyroly-

50 tic graphite electrodes modified with layers of multiwalled carbon nanotubes. ${ }^{43}$ A gradient of 0.69 was obtained, which is indicative of a possible mixed mass transport regime with thinlayer diff usion within the porous conductive layer, and semiinfinite diff usion outside the layer in solution. ${ }^{43}$ Paper is also a porous material with channels and pores of variable dimensions and mixed behavior can be observed.

On the other hand, the electrochemical behavior of AA on PCWEs fabricated with 25 and $50 \%$ carbon ink suspensions
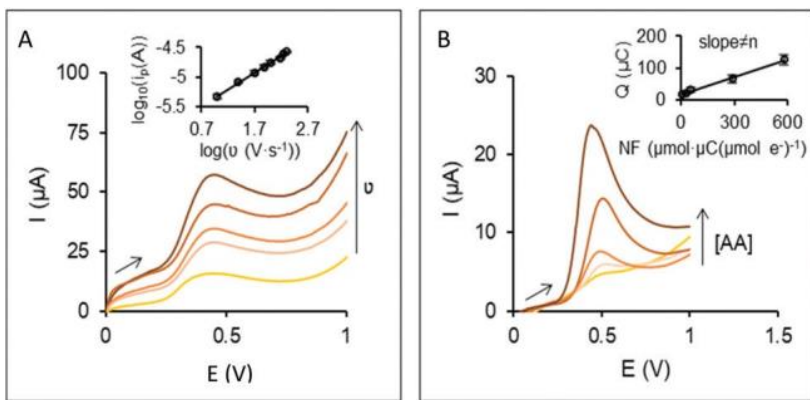

1

Fig. 2 (A) Linear sweep voltammograms recorded in $1 \mathrm{mM}$ AA solutions (in acetate buff er pH 3.5) at 10, 25, 50, 100, and $200 \mathrm{mV} \mathrm{s}^{-1}$. Inset: plot of the logarithm of the peak current $\left(i_{p}\right) v s$. the logarithm of $v$. Error bars correspond to the standard deviation of the measurements obtained in diff erent PCWEs $(n=3)$. (B) Linear sweep voltammograms recorded 15 using PCWEs with $25 \%$ carbon ink in solutions of diff erent concentrations of $\mathrm{AA}$ ( $\mathrm{pH}$ 3.5). Inset: $\mathrm{Q}$ vs. NF plot. Scan rate: $100 \mathrm{mV} \mathrm{s}^{-1}$. Error bars correspond to the standard deviation of the measurements obtained in diff erent PCWEs $(n=3)$.

(in DMF) as well as with undiluted ink was compared. The eff ect of the carbon concentration was evaluated by recording LSVs in AA solutions of diff erent concentrations, in a range between 0.01 and $1.00 \mathrm{mM}$ in acetate buff er, pH 3.5. The 25 results obtained from the $i_{\mathrm{p}} v S$. $C$ plots (Fig. S1†) showed that the sensitivity decreases with the concentration of carbon

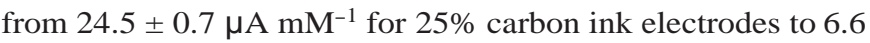
$\pm 0.8 \mu \mathrm{A} \mathrm{mM}^{-1}$ for $100 \%$ carbon ink electrodes. Linearity and precision are notoriously better for the suspension with $25 \%$ carbon ink. Diff erent electroactive areas of the PCWEs will probably be the reason for this diff erence.

The ratio between the electrochemical active areas can be estimated from the slopes of the $i_{\mathrm{p}} v s$. $C$ representation (Fig. S2†). Regardless of the mass-transport regime, the peak 35 current is proportional to the electroactive area and the concentration of the species in solution. Since all the parameters, except the electroactive area, remain constant for all the PCWEs used in this work, the ratios between the slopes for 40 two PCWEs made of diff erent carbon ink concentrations give directly the ratios between their areas. Then, lower area corresponds to undiluted carbon ink PCWEs. Diluted suspensions could diff use better through the paper, filling the pores and

increasing the active area. The area proportionated by $25 \% 45$ carbon ink suspensions is 1.7 and 3.7 times higher than that obtained using 50 and $100 \%$ carbon ink, respectively. Higher dilution did not increase the signal probably due to lower interconnection between more dispersed carbon particles. Then, a $25 \%$ carbon ink suspension was chosen for the rest of the work. The design of the electrochemical cell is very convenient due to several advantages, mainly (i) simplicity: there is no need to use screen-printing since a carbon ink suspension can be deposited with the help of a micropipette on a delimited working hydrophilic area; (ii) bifunctionality: the 55 paper acts as a container of both the conductive ink and the sample; (iii) low-cost: apart from the paper-based component (where the deposition of the ink avoids ink waste), the cell is 
1 completed with commercial electronic connectors. Moreover, low sample volumes are employed ( $\mu \mathrm{L}$ ); (iv) fast response: the analyte is rapidly electrolysed with efficient mass transport to the electrode surface (thin-layer samples); (v) portability: the small size and the low number of components (paper/ink and connectors) make the platforms easy-to-transport and (vi) disposability/reusability: although the paper platform is disposed of after measurement, the gold-plated connectors can be reused for following measurements with readily replaced 10 paper electrodes.

Regarding the technique, voltammograms have the benefit of providing peak-shaped responses and can be better analyzed than the monotonous current decays observed with the application of a constant potential. However, more complicated instrumentation is required for LSV measurements because a potential scan with controlled scan rate is performed. Meanwhile, chronoamperometric measurements only require the application of a constant potential, which could be 20 provided by using a simple battery.

Fig. 2B shows experimental voltammograms for diff erent AA concentrations recorded in acetate buff er $\mathrm{pH} 3.5$ with well- defined peaks. The integrated charge corresponding to LSV peaks recorded at $100 \mathrm{mV} \mathrm{s}^{-1}$ was found to be linear in the

0.01-1 $\mathrm{mM}$ concentration range and followed the equation $Q=$ $(110 \pm 4.3) C \mu \mathrm{C} \mathrm{mM}{ }^{-1}+(16 \pm 2.2) \mu \mathrm{C}$, with $R^{2}=0.996$. Good precision was obtained using three PCWEs for each concentration. The limit of detection (LOD) was $20 \mu \mathrm{M}$, calculated as $3 S_{\mathrm{a}} / m$ where $m$ and $S_{\mathrm{a}}$ are the slope and the deviation of the intercept of the $Q v s$. C calibration curve (not shown). To be an absolute method and according to Faraday's law, the slope of the $Q v s$. NF plot should have the same value as the number of transferred electrons. In this case the plot, shown in the inset of Fig. 2B, shows that Faraday's law is not fulfilled since the value of the slope is $0.19 \pm 0.01$ moles of electrons per mol of AA, one order lower than $n$. If the experimental $n$ value had matched $2 \mathrm{e}^{-}$, a calibration curve would not be required. These results indicate that probably the scan rate $\left(100 \mathrm{mV} \mathrm{s}^{-1}\right)$ pro-

vides too low time (ca. $2 \mathrm{~s}$ calculated from the peak width) to deplete the AA. Lower scan rates off er higher times but also

decrease in the faradaic current (Fig. 2A).

Although LSV can be employed for AA determination using an external calibration curve ( $Q$ or $I v s . C$ plot), its determination by controlled-potential coulometry, a calibration-free methodology, was evaluated.

Determination of $\mathrm{L}_{\mathrm{L}}$-ascorbic acid by controlled-potential coulometry

50 Once the electrochemical behavior of AA has been explored by linear sweep voltammetry on paper-based electrodes, no further optimization was required to perform the coulometric determination of AA. This design increases the coulometric efficiency, related to the transfer of electrons in a fast and eff ective way, when compared to traditional schemes. There are several points responsible for this: (i) fast passive transport by gravity and capillary forces to the porous electrode situated at the bottom, (ii) high electrode area with conductive ink that fills the pores (on one side) of the paper when the ink is deposited, and (iii) generation of a thin layer of liquid over the electrode surface that is integrated with the cellulosic micro and nanofibers.

The total charge calculated as the area under the curve of chronoamperograms, acquired for diff erent concentrations of AA in acetate buff er pH 3.5, constitutes the analytical signal. Double-step chronoamperometry was carried out applying a first potential where redox processes do not take place in order to get a stable baseline. Then, $+0.0 \mathrm{~V}$ was applied for $10 \mathrm{~s}$ followed by the application of $+0.6 \mathrm{~V}$ for $50 \mathrm{~s}$, enough potential for AA oxidation. Fig. 3A shows the chronoamperograms obtained in $1 \mathrm{mM}$ AA solutions in acetate buff er $\mathrm{pH} 3.5$ for three diff erent PCWEs. Good precision was obtained, with an integrated charge value of $1.21 \pm 0.06 \mathrm{mC}$. In addition, reordering Faraday's Law the calculation of $Q / N F$ (where $N$ is $6 \times 10^{-3}$ mmol) gave as a result a value of $2.1 \pm 0.1$ moles of electrons per mol of AA (number of electrons). This suggests that the method could be suitable for calibration-free determination 20 since this value coincides with the reported number of electrons transferred $\left(2 \mathrm{e}^{-}\right)$.

In order to demonstrate that Faraday's law is accomplished in a convenient interval of AA concentrations (fruit juices contain 6-10 mg of AA per $100 \mathrm{~mL}$, equivalent to a 25 $0.35-0.57 \mathrm{mM}$ concentration interval), chronoamperometry was carried out in AA solutions prepared in acetate buff er $\mathrm{pH}$ 3.5, with diff erent concentrations ranging from 0.05 to $1 \mathrm{mM}$. Chronoamperograms are shown in Fig. 3B. In the inset, the corresponding area under the graph $(Q)$ is represented $v s . \mathrm{NF}, 30$ showing a linear relationship. The error bars indicate the standard deviation of the measurements, performed in triplicate. The average of the corresponding relative standard deviations is $9.6 \%$, which indicates adequate precision, in terms repeatability, of the methodology. Apart from this, the reproducibility of these paper devices had been previously studied for glucose determination using a bienzymatic methodology with electron transfer mediation. ${ }^{30}$ In that case the value of the RSD for diff erent devices (diff erent WEs, REs and AEs) and a $2.5 \mathrm{mM}$ glucose concentration was below $5 \%$. The slope of the

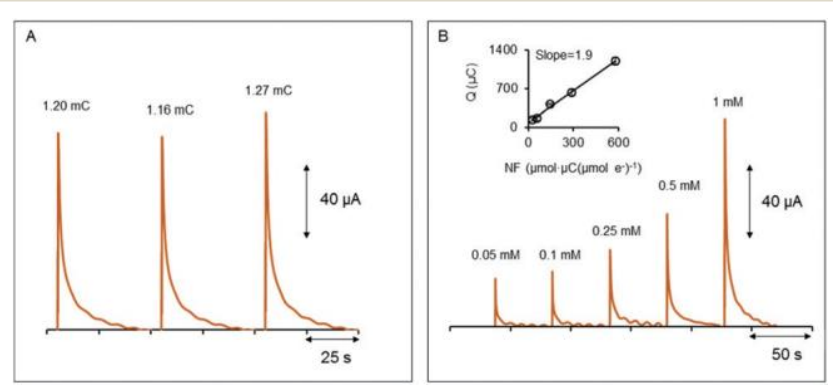

Fig. 3 Chronoamperograms recorded in acetate buff er $-\mathrm{pH} 3.5$ with $1 \mathrm{mM}(\mathrm{A})$ and diff erent concentrations of AA ranging from 0.05 to $1 \mathrm{mM} 55$ (B). Inset: $Q$ vs. NF plot where $Q$ is the charge obtained by measuring the area under the chronoamperograms. Error bars correspond to the standard deviation of measurements performed in several PCWEs $(n=3)$. 
1 representation, with a value of $1.9 \pm 0.1$ moles of $\mathrm{e}^{-}$per mol of AA, confirms the accomplishment of Faraday's law with an $R^{2}$ of 0.996 and a limit of detection (LOD) of $0.05 \mathrm{mM}$, calculated as $3 S_{\mathrm{a}} / m$ where $m$ and $S_{\mathrm{a}}$ are the slope and the standard deviation of the intercept respectively, of the $Q v s$. $C$ calibration curve (not shown). The determination of AA can also be carried out using the calibration plot $i v s$. $C$, measuring the current at a fixed time in chronoamperograms recorded for diff erent concentrations of AA (Fig. S3†) but the main goal of

10 this work is the achievement of a method which does not require calibration, minimizing cost and time analysis.

Although in some cases evaporation can be a useful strategy (as a preconcentration $\mathrm{step}^{44}$ ), in most cases it is one of the main concerns of paper-based devices. Concentration-dependent detectors can see their precision reduced due to the evaporation of the solvent in liquid samples. An electrode with a high surface-to-volume ratio is very convenient for favoring exhaustive electrolysis. However, solvent evaporation can be an important side eff ect, which provokes a consequent increase in the concentration. In these cases, strategies such as sealing the paper with an adhesive tape ${ }^{45}$ or enclosing it in a plastic holder ${ }^{5}$ could be utilized. In any case, since coulometry is a mass-dependent technique, with the charge related to the number of moles electrolyzed through the Faraday equation, it is very convenient when evaporation is possible. Moreover, solutions are not aspirated by capillarity but deposited on the paper and the electrolysis time (60 s of measurement, with most of the analyte electrolyzed after approximately $30 \mathrm{~s}$, as can be seen in Fig. 3) is lower than in traditional coulometry, which minimizes evaporation. These factors are important to ensure the accuracy of the devices, since the mass is maintained even when the volume could vary slightly.

Ascorbic acid has been chosen in many cases as a model analyte to demonstrate the utility of diff erent designs of paper devices. Although in most of the cases clinical samples are analysed, designs can be translated to the agri-food sector. Thus, AA can be determined in commercial tablets using pencil drawn electrodes in an area delimited by laser-printed toner lines. ${ }^{46}$ Using these devices and voltammetric techniques, a linear behavior was obtained between 0.5 and $3.0 \mathrm{mM}$ (LOD: $0.07 \mathrm{mM}$ ). Screen-printing and inkjet-printing can be combined for depositing a base material (carbon) and a modifying functional material ( polyaniline) on a filter paper. ${ }^{47}$ The limit of detection in this case is $0.03 \mathrm{mM}$. In the area of food surveillance, paper-based devices are very promising. One of the main advantages is the versatility: both hydrophilic and hydrophobic cellulosic substrates could be employed. ${ }^{48}$ One

50 example of the last one is a device constructed using the paper employed in printed electronics and inkjet-printed silver and carbon inks. A dispersion based on carbon black nanoparticles can be used to modify the electrochemical sensor platform and decrease the limit of detection of AA (from 0.25 to

$0.15 \mathrm{mM}$ ), determined in dietary supplements by cyclic voltammetry. ${ }^{49}$ The value agreed with that reported on the label. Apart from this, porous paper allows performing not only static but also flow determinations. In the last case, fluids can be driven by both hydrostatic and capillary forces. Origami folding paper (oPAD) and a movable reagent-stored platform (rPAD) were employed in a flow-through configuration for AA determination in vitamin $\mathrm{C}$ juices. ${ }^{50} \mathrm{~A}$ linear range was obtained between 0.15 and $0.8 \mathrm{mM}$ (LOD: $0.093 \mathrm{mM}$ ), and the values obtained for real sample analysis agreed also with those reported in the label. In our case, simplicity is the main advantage over those diff erent and interesting examples commented, since (i) electrodes are easily fabricated without requiring screens or stencils. Deposition of the ink from a micropipette is a way to fabricate the working electrode precisely. In this case it is not modified but it could be done previously or when the ink has been already deposited. The reference and auxiliary electrodes are the pins of a connector header, which is given a new function and can be reused, (ii) common hydrophilic chromatography paper is employed as the electrochemical cell, to contain the electrodes, electrolyte and analyte, and (iii) a single-use static device is employed. However, if several measurements need to be performed in a fast way, instead of using sequential measurements (as in a flow configuration), they could be performed simultaneously in a multiplexed design. ${ }^{51}$ This design allows performing diff erent techniques, in this work: cyclic voltammetry, chron-

oamperometry and coulometry, the last one employing a calibration-free methodology. The limit of detection was $0.05 \mathrm{mM}$, which is in the same range (in most cases even below) as those reported in the examples.

As an absolute method, the concentration of AA in real samples can be determined by mere calculation of the reordered Faraday's law $N=Q / n F$. Considering that $N=C \times V$ (where $V$ is the volume of the sample), the concentration is given by the formula $C=Q / n F V$. In this case, since the volume is $6 \times 10^{-6} \mathrm{~L}, C(\mathrm{mM})=0.86\left(\mathrm{mM} \cdot \mathrm{mC}^{-1}\right) Q(\mathrm{mC})$. Three chronoamperometric measurements in $6 \mu \mathrm{L}$ aliquots of the sample are then enough for the determination.

\section{Calibration-free determination of ascorbic acid in real samples}

Real sample analysis of AA was performed for diff erent fruit juices (orange, apple and multi-fruit). The only pretreatment required was dilution with buff er to be included in the dynamic linear range. A limitation of the coulometric method is related to the $100 \%$ current efficiency requirement, i.e. the selectivity. If substances that can be electrolyzed are present in 45 the sample (e.g. hydrogen peroxide) the current is not going to be only due to AA oxidation and the results will not be accurate. In any case, a cyclic voltammogram can be recorded to observe the electrochemical processes that can be involved and then the possible interferences. Modifications of the working 50 electrode can be made in order to diff erentiate between molecules with close redox potentials. ${ }^{52}$ The strategy employed here to solve this was the use of ascorbate oxidase to oxidize Lascorbic acid and convert it into dehydro-L-ascorbate. ${ }^{53}$ In this way, a background signal can be obtained. This step has 55 been made off -line, as a previous pretreatment. An integrated step, with the enzyme stored on paper, could be an interesting approach. 


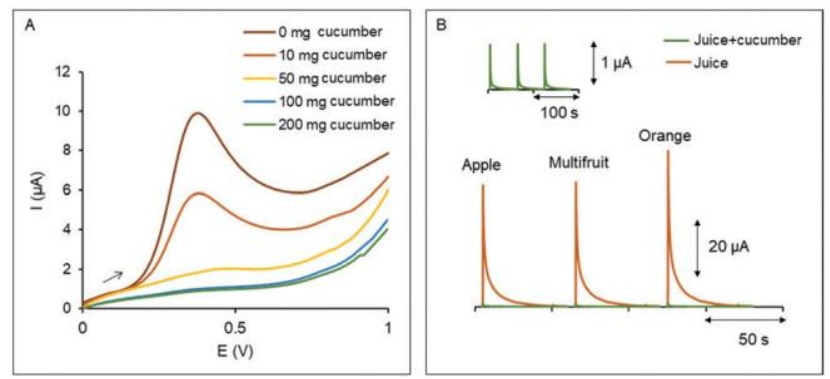

Fig. 4 (A) Linear sweep voltammograms recorded in enzyme-treated and untreated $0.5 \mathrm{mM}$ AA solutions in acetate buff er -pH 5.0. Samples were then diluted $1: 1$ with acetate buff er $\mathrm{pH}$ 3.5. (B) Chronoamperograms recorded in enzyme-treated and untreated sample solutions in acetate buff er $-\mathrm{pH} 5.0$, diluted $1: 1$ with acetate buff er pH 3.5. Inset: zoom of signals obtained after the enzymatic treatment of juice samples.

Then, to eliminate the influence of the matrix on the response of the PCWEs for ascorbic acid, fruit juice samples were measured before and after enzymatic treatment with cucumber L-ascorbate oxidase. The cucumber Lascorbate oxidase contains 8 atoms of copper per mole-

5 cular weight of $132 \mathrm{kDa}$ and has a specific activity of $3500 \mathrm{U} \mathrm{mg}^{-1}$. $54,55,56$

Studies were performed by LSV to know the lowest amount of cucumber peel required to deplete all AA in solution. Measured solutions were the result of mixing 10-200 mg of cucumber peel with $0.5 \mathrm{mM}$ AA solutions in acetate buff er $\mathrm{pH}$ 5.0, subsequently diluted with acetate buff er $\mathrm{pH}$ 3.5 .

Voltammograms in Fig. 4A show the eff ectiveness of ascorbate oxidase in eliminating ascorbic acid from the solution, as indi- cated in Scheme S2. $†$

Total suppression of the AA signal is observed when $100 \mathrm{mg}$ of cucumber peel were added and no diff erences are observed for higher amounts of cucumber.

Therefore, real samples were mixed with $100 \mathrm{mg}$ of cucumber according to the procedure described in the Experimental section. In Fig. 4B the chronoamperograms recorded in diluted juices, before and after the pretreatment, are shown and the results indicate that there are no matrix interferences. This fact is confirmed by cyclic voltammograms recorded in pretreated and untreated sample solutions (Fig. S4†).

Table 1 shows the results obtained for AA determination together with the nominal values reported on the label. Although the comparison with a reference methodology would

Table 1 Results for AA determination obtained in fruit juices by the calibration-free method and nominal values

\begin{tabular}{lll} 
Juice & $\begin{array}{l}\text { Calibration-free } \\
\text { coulometric method } \\
{[\mathrm{AA}](\mathrm{mg} \text { per } 100 \mathrm{~mL})}\end{array}$ & $\begin{array}{l}\text { Value declared } \\
\text { in the fruit } \\
{[\mathrm{AA}](\mathrm{mg} \text { per } 100 \mathrm{~mL})}\end{array}$ \\
\hline Apple & $5.5 \pm 0.6$ & 6.0 \\
Multi-fruit & $5.9 \pm 0.6$ & 6.0 \\
Orange & $9.6 \pm 0.4$ & 10.0
\end{tabular}

also have been very adequate, ascorbic acid was chosen as a model analyte to demonstrate the suitability of the coulometric readout in paper-based devices. Thus, the results were compared with the value declared in the label as in other ascorbic acid determinations in food samples. ${ }^{49,50}$ The charges calculated in enzyme-treated samples were subtracted from those obtained in untreated samples in order to calculate the charge due only to the presence of AA in the diff erent

juices. The values of the relative standard deviation are 10.2, 10.6 and $4.2 \%$ for the apple, multi-fruit and orange juice samples respectively. The application of Student's t-test demonstrated that there were no significant diff erences between the nominal value and those obtained by the calibration-free method on paper at a 0.05 significance level when apple and multi-fruit juices were analyzed. This indicates the good accuracy and precision achieved, even for colored and turbid samples.

\section{Conclusions}

We have presented a promising controlled-potential coulometric method using paper-based electrodes for the point-ofneed determination of AA. The simple calculation of the total charge by integrating the area under the curve of a chronoamperogram and the low volume required $(6 \mu \mathrm{L})$ provide a fast and accurate calibration-free method. This methodology reduces enormously the cost of determinations performed with more common electrochemical techniques, such as CV, because calibrators are not needed and the time of analysis is considerably reduced. Since coulometry is performed at a constant potential the equipment can also be simplified.

In addition, the platform employed is the combination of reusable gold-plated connector headers with disposable paper electrodes modified with $25 \%$ carbon ink suspensions and provides reproducible, low-cost, simple, and portable devices, suitable for decentralized analysis. We have demonstrated its potential for the AA determination in real food samples with good accuracy. Matrix interferences were studied by depleting AA with ascorbate oxidase provided by a natural source, the cucumber, low-cost vegetable available in any grocery. Samples and sample-cucumber treated mixtures were directly measured after their dilution, demonstrating the simplicity, user friendliness, easy handling and great performance of the absolute method. Incorporation of diff erent steps will simplify even more the methodology and advance towards real lab-on-paper devices. Integration of the sampling step using a microfluidic "tongue" has already been demonstrated. ${ }^{51}$ Work is in progress to incorporate dilution, the only pretreatment required for these liquid samples.

\section{Conflicts of interest}

There are no conflicts to declare. 


\section{Acknowledgements}

This work was supported by the Spanish Ministry of Economy and Competitiveness (MINECO) under project CTQ2014-58826-

R. Estefania Núnez-Bajo thanks MINECO for the award of a PhD grant BES-2012-056713.

\section{References}

1 T. Gebretsadik, T. Belayneh, S. Gebremichael, W. Linert, M. Thomas and T. Berhanu, Analyst, 2019, 144, 2467-2479.

2 E. Noviana, C. P. McCord, K. M. Clark, I. Jang and C. S. Henry, Lab Chip, 2020.

3 J. Adkins, K. Boehle and C. S. Henry, Electrophoresis, 2015, 36, 1811-1824.

4 N. F. D. Silva, C. M. R. Almeida, J. M. C. S. Magalhaes, M. P. Goncalves, C. Freire and C. Delerue-Matos, Biosens. Bioelectron., 2019, 141, 111317.

5 E. Núnez-Bajo, M. C. Blanco-López, A. Costa-García and M. T. Fernández-Abedul, Biosens. Bioelectron., 2017, 91, 824-832.

6 J. J. Lingane, J. Am. Chem. Soc., 1945, 67, 1916-1922.

7 A. J. Bard, Anal. Chem., 1963, 35, 1125-1128.

8 A. J. Bard, Anal. Chem., 1968, 40, 64R.

9 M. Cuartero, G. A. Crespo and E. Bakker, Anal. Chem., 2015, 87, 1981-1990.

10 E. Bakker, Can calibration-free sensors be realized?, ACS Sens., 2016, 1, 838-841.

11 A. W. Martinez, S. T. Phillips, M. J. Butte and G. M. Whitesides, Angew. Chem., Int. Ed., 2007, 46, 13181320.

12 W. Dungchai, O. Chailapakul and C. S. Henry, Anal. Chem., 2009, 81, 5821-5826.

13 M. M. Hamedi, A. Ainla, F. Güder, D. C. Christodouleas, M. T. Fernández-Abedul and G. M. Whitesides, Adv. Mater., 2016, 28, 5054-5063.

14 A. M. Pisoschi, M. C. Cheregi and A. F. Danet, Molecules, 2009, 14, 480-493.

15 J. Zak and T. Kuwana, J. Am. Chem. Soc., 1982, 104, 55145515.

16 S. Dong and T. Kuwana, J. Electrochem. Soc., 1984, 131, 813-819.

17 P. Karabinas and D. Jannakoudakis, J. Electroanal. Chem. Interfacial Electrochem., 1984, 160, 159-167.

18 I.-F. Hu, D. H. Karweik and T. Kuwana, J. Electroanal. Chem. Interfacial Electrochem., 1985, 188, 59-72.

19 J. Kulys and E. J. D’Costa, Anal. Chim. Acta, 1991, 243, 173178.

20 A. M. Pisoschi, A. Pop, A. I. Serban and C. Fafaneata, Electrochim. Acta, 2014, 121, 443-460.

21 S. Skrovankova, J. Mlcek, J. Sochor, M. Baron, J. Kynicky and T. Jurikova, Int. J. Electrochem. Sci., 2015, 10, 24212431.

22 F. Wu, T. Huang, Y. Hu, X. Yang, Y. Ouyanga and Q. Xie, Microchim. Acta, 2016, 183, 2539-2546.
23 E. Luque-Perez, A. Rios and M. Valcarcel, Fresenius' J. Anal. Chem., 2000, 366, 857-862.

24 M. Özyürek, K. Güçlü, B. Bektaşoğlu and R. Apak, Anal. Chim. Acta, 2007, 588, 88-95.

25 J. J. Liu, Z. T. Chen, D. S. Tang, Y. B. Wang, L. T. Kang and J. N. Yao, Sens. Actuators, B, 2015, 212, 214221.

26 T. Pérez-Ruíz, C. Martínez-Lozano and A. Sanz, Anal. Chim. Acta, 1995, 308, 299-307.

27 T. R. L. C. Paixao, D. Lowinsohn and M. Bertotti, J. Agric. Food Chem., 2006, 54, 3072-3077.

28 S. Harris, J. Gonzales, S. Melaku and R. B. Dabke, ACS Omega, 2019, 4, 3684-3689.

29 B. Liang, Q. Zhu, L. Fang, Q. Cao, X. Liang and X. Ye, Electrochem. Commun., 2017, 82, 43-46.

30 O. Amor-Gutiérrez, E. Costa-Rama, A. Costa-García and M. T. Fernández-Abedul, Biosens. Bioelectron., 2016, 93, 40-45.

31 A. de Donato, J. J. Pedrotti and I. G. R. Gutz, Electroanalysis, 1999, 11, 1124-1129.

32 W. Y. Chung, J. K. O. Chung, Y. T. Szeto, B. Tomlinson and I. F. Benzie, Clin. Biochem., 2001, 34, 623-625.

33 L. Nováková, P. Solich and D. Solichová, TrAC, Trends Anal. Chem., 2008, 27, 942-958.

34 H. A. Dabbagh, F. Azami, H. Farrokhpour and A. N. Chermahini, J. Mol. Struct., 2014, 1061, 69-75.

35 H. Remini, C. Mertz, A. Belbahi, N. Achir, M. Dornier and K. Madani, Food Chem., 2015, 173, 665-673.

36 R. N. Adams, Anal. Chem., 1976, 48, 1126A-1138A.

37 X. L. Wen, Z. X. Han, A. Rieker and Z. L. Liu, J. Chem. Res., 1997, 3, 108-109.

38 C. S. Erdurak-Kilic, B. Uslu, B. Dogan, U. Ozgen, S. A. Ozkan and M. Coskun, Anal. Chem., 2006, 61, 1113-1120.

39 S. S. Shankar, B. E. K. Swam, B. N. Chandrashekar and K. J. Gururaj, J. Mol. Liq., 2013, 177, 32-39.

40 M. Rueda, A. Aldaz and F. Sanchez-Burgos, Electrochim. Acta, 1978, 23, 419-424.

41 S. S. Kumar, J. Mathiyarasu, K. L. N. Phani and V. Yegnaraman, J. Solid State Electrochem., 2006, 10, 905913.

42 A. J. Bard and L. R. Faulkner, Electrochemical Methods Fundamentals and Applications, Wiley, New York, 2000.

43 M. J. Sims, N. V. Rees, E. J. F. Dickinson and R. G. Compton, Sens. Actuators, B, 2010, 144, 153-158.

44 E. Costa-Rama, H. P. A. Nouws, C. Delerue-Matos, M. C. Blanco-López and M. T. Fernández-Abedul, Anal. Chim. Acta, 2019, 1074, 89-97.

45 A. Pal, H. E. Cuellar, R. Kuang, H. F. N. Caurin, D. Goswami and R. V. Martínez, Adv. Mater. Technol., 2017, 2, 1700130.

46 V. X. G. Oliveira, A. A. Dias, L. L. Carvalho, T. M. G. Cardoso, F. Colmati and W. K. T. Coltro, Anal. Sci., 2018, 34, 91-95.

47 W. Kit-Anan, A. Olarnwanich, C. Sriprachuabwong, C. Karuwan, A. Tuantranont, A. Wisitsoraat, W. Srituravanich and A. Pimpin, J. Electroanal. Chem., 2012, 685, 72-78. 
148 S. Cinti, Anal. Bioanal. Chem., 2019, 411, 4303-4311.

49 S. Cinti, N. Colozza, I. Cacciotti, D. Moscone, M. Polomoshnov, E. Sowade, R. R. Baumann and F. Arduini, Sens. Actuators, B, 2018, 265, 155160.

50 A. Yakoh, S. Chaiyo, W. Siangproh and O. Chailapakul, ACS Sens., 2019, 4, 1211-1221.

51 O. Amor-Gutiérrez, E. Costa-Rama and M. T. FernándezAbedul, Biosens. Bioelectron., 2019, 135, 64-70.
52 B. Dinesh, R. Saraswathi and A. S. Kumar, Electrochim. 1 Acta, 2017, 233, 92-104.

53 E. Akyilmaz and E. Dinckaya, Talanta, 1999, 50, 87-93.

54 K. Kawahara, S. Suzuki, T. Sakurai and A. Nakahara, Arch. Biochem. Biophys., 1985, 241, 179-186.

55 T. Nakamura, N. Makino and Y. Ogura, J. Biochem., 1968, 64, 189-195.

56 T. Sakura, S. Sawara, S. Nakahara and A. Suzuki, Biochem. Biophys. Res. Commun., 1985, 131, 647-652. 